\title{
Noninvasive Estimation of Myosin Heavy Chain Composition in Human Skeletal Muscle
}

\author{
BOŠTJAN ŠIMUNIČ ${ }^{1}$, HANS DEGENS ${ }^{2}$, JÖRN RITTWEGER ${ }^{2,3}$, MARCO NARICI $^{2}$, IGOR B. MEKJAVIĆ ${ }^{4}$, \\ and RADO PIŠOT ${ }^{1}$ \\ ${ }^{1}$ Institute for Kinesiology Research, Science and Research Centre Koper, University of Primorska, Koper, SLOVENIA; \\ ${ }^{2}$ Institute for Biomedical Research into Human Movement and Health, Manchester Metropolitan University, Manchester, \\ UNITED KINGDOM; ${ }^{3}$ Institute of Aerospace Medicine, German Aerospace Center, Cologne, GERMANY; \\ and ${ }^{4}$ Department of Automation, Biocybernetics, and Robotics, Jožef Stefan Institute, Ljubljana, SLOVENIA
}

\begin{abstract}
ŠIMUNIČ, B., H. DEGENS, J. RITTWEGER, M. NARICI, I. B. MEKJAVIĆ, and R. PIŠOT. Noninvasive Estimation of Myosin Heavy Chain Composition in Human Skeletal Muscle. Med. Sci. Sports Exerc., Vol. 43, No. 9, pp. 1619-1625, 2011. Purpose: Information on muscle fiber type composition is of great importance in muscle physiology and athletic performance. Because there are only a few techniques available that noninvasively and accurately provide an estimate of muscle fiber type composition, the development of additional and alternative approaches is required. Methods: Twenty-seven participants (21 men, 6 women) with an average age of $43 \pm$ $18 \mathrm{yr}$, height of $175 \pm 7 \mathrm{~cm}$, and mass of $74 \pm 12 \mathrm{~kg}$ participated in the study. Delay, contraction, and half relaxation times were calculated from tensiomyographic radial twitch responses of the vastus lateralis muscle. Univariate and multiple linear regression analyses were used to correlate the proportion of myosin heavy chain I (\%MHC-I) in a biopsy obtained from the same muscle with a single and all three radial twitch parameters. Results: Delay, contraction, and half relaxation times all correlated with \%MHC-I $(r=0.612,0.878$, and 0.669 , respectively, at $P \leq 0.001$ ). When all three parameters were included in a multiple linear regression, the correlation with the \%MHC-I was even better $(R=0.933, P<0.001)$. Conclusions: These data suggest that time parameters of the skeletal muscle mechanical radial twitch response, measured with a contact linear displacement sensor, can be used as an accurate noninvasive predictor of the \%MHC-I in a muscle. Key Words: TENSIOMYOGRAPHY, FIBER TYPE, MYOSIN HEAVY CHAIN, MECHANOMYOGRAPHY
\end{abstract}

$\mathrm{T}$ There is considerable interest in the fiber type composition of a muscle, particularly for researchers and also for athletes. Researchers are interested in training and aging effects and also wish to characterize test subjects. Although there is still uncertainty as to what extent the fiber type composition is predictive of athletic performance $(19,39)$, human muscle power is mainly determined by the fiber type composition of the protagonist muscles, the muscular strength/mass, and the level of the neuromuscular activation during the movement (23). This corresponds with the higher power-generating capacity of the fast type II muscle than of the slow type I fibers (14), which are, however, more fatigable (32). An invasive muscle biopsy is used also for muscle fiber type determination, and there is a substantial need to develop a valid and reliable noninvasive methodology for fiber type estimation from biomechanical muscle response.

\footnotetext{
Address for correspondence: Boštjan Šimunič, UP-ZRS, Garibaldijeva 1, 6000 Koper, Slovenia; E-mail: Bostjan.simunic@zrs.upr.si.

Submitted for publication September 2010.

Accepted for publication February 2011.

0195-9131/11/4309-1619/0

MEDICINE \& SCIENCE IN SPORTS \& EXERCISE $E_{\circledast}$

Copyright $(\subset) 2011$ by the American College of Sports Medicine

DOI: 10.1249/MSS.0b013e31821522d0
}

Delay time $(\mathrm{Td})$, contraction time $(\mathrm{Tc})$, and half relaxation time (Tr) are biomechanical parameters of skeletal muscle contraction $(4-5,7)$. Contraction time is defined as the time interval between the onset of development of twitch force and its peak and thus reflects the speed of force generation. This parameter readily distinguishes slow and fast motor units and muscles $(5,7)$. Muscle twitch force/ torque responses invoked by an electrical stimulus are frequently used to analyze muscle contractile properties $(6,8-9$, $12,16,17)$. There are many factors that clearly make the interpretation of the force/torque twitch Tc of a muscle difficult, if not impossible: the force/torque twitch amplitude is of a low amplitude, typically lower than 10\% MVC (34), and has to be transmitted through connective tissue to be registered by an external force transducer; the degree of slack of the muscle and the compliance of both connective tissue and muscle affect the shape of the twitch; the force/ torque response integrates responses of synergistic and antagonistic muscle heads because of cross talk between adjacent muscles (18); and the force/torque response is delayed because of mechanical damping of the active muscle tissue by the passive surrounding tissue (37).

To avoid or minimize the factors mentioned above, different approaches are proposed that measure muscle deformation during a twitch in an alternative transversal (radial) plane $(1,10,22,27-30,33,36,40,44)$. Because muscle volume does not change during a contraction $(16,38)$, length 
changes must be accompanied by proportional changes in the width of the muscle. Several mechanomyographic methods have been proposed that are based on measuring lateral vibrations and thickening of the muscle during a contraction. For instance, in phonomyography (22) or soundmyography $(1,30)$, microphones are used to transform muscle fiber mechanical oscillations at its resonance frequency into audible sound, whereas in vibromyography (44), accelerometers and laser beams are used (27-29) to detect thickening and vibration of a whole muscle belly. Promising results have been obtained using mechanomyographic methods, but there are several difficulties $(26,43)$, such as a low signal-to-noise ratio and consequently high variability, complex measuring setup and/or expensive hardware, and necessary postprocessing of the signals.

Another method is tensiomyography, which requires a relatively simple setup and detects radial muscle belly enlargement using a contact displacement sensor $(10,20,33$, $36,40)$. This technique has been suggested to provide valuable information regarding muscle contraction characteristics $(10,40)$, and indeed, Dahmane et al. (10) found a positive correlation between the radial Tc of seven different muscles in vivo and the proportion of type I fibers in biopsies from corresponding muscles in cadavers. Although the correlation by Dahmane et al. (10) was significant, the spread of the data was too high for practical use. A comparison between tensiomyography and the proportion of myosin heavy chain I (\%MHC-I) of the very same muscle has not been done.

Therefore, the aim of our study was to evaluate the correlation between radial twitch $\mathrm{Td}, \mathrm{Tc}$, and $\mathrm{Tr}$ measured by tensiomyography and \%MHC-I in the vastus lateralis muscle. Furthermore, we used a multiple regression analysis of $\%$ MHC-I versus tensiomyographically determined radial twitch Td, Tc, and $\mathrm{Tr}$ to establish noninvasive estimators of \%MHC-I in human skeletal muscle.

\section{MATERIALS AND METHODS}

Participants. Twenty-one males (age $=45 \pm 19$ yr, ranged from 20 to $83 \mathrm{yr}$; height $=177 \pm 6 \mathrm{~cm}$; mass $=76 \pm$ $9 \mathrm{~kg}$ ) and six females (age $=51 \pm 10 \mathrm{yr}$, ranged from 36 to $66 \mathrm{yr}$; height $=168 \pm 4 \mathrm{~cm}$; mass $=62 \pm 7 \mathrm{~kg}$ ) without any history of neuromuscular and cardiovascular disorders gave written informed consent to participate in this study. Participants were recruited during two studies. Study 1 was a bed rest study carried out in Valdoltra, Slovenia, in 2007. During study 1, tensiomyography was performed, and biopsies were obtained from the vastus lateralis muscle in 10 male fit participants (average relative oxygen consumption $=51 \pm 8 \mathrm{~mL} \cdot \mathrm{min}^{-1} \cdot \mathrm{kg}^{-1}$, average countermovement jump height $=49 \pm 5 \mathrm{~cm}$ ). Study 2 was a large-scale study into neuromuscular performance in master athletes during the 16th European Veterans Athletic Championships in Ljubljana, Slovenia, in 2008. In the context of this study, 11 male and 6 female participants were involved for biopsy and the tensiomyography of the vastus lateralis muscle. Of the master athletes, 10 were anaerobic athletes (6 throwers, 3 jumpers, 1 sprinter), 5 were aerobic athletes ( 3 steeplechasers, 2 runners), and 2 were decathletes. All participants gave their written consent to participate in the studies. All procedures conformed to the 1964 Declaration of Helsinki and were approved by the Committee for Medical Ethics at the Ministry of Health (Slovenia).

Experimental design. All testing procedures were explained to the participants. A biopsy was taken from and tensiomyographic measurements were made on the right vastus lateralis muscle.

In study 1, all 10 biopsies were collected at the initial day of bed rest when non-high-intensity exercise was performed. The tensiomyographic data were collected on the same day approximately $1 \mathrm{~h}$ before the biopsy. The day before, all participants performed a submaximal aerobic performance test on a cycle ergometer. None of the participants complained about muscle pain and general or peripheral exhaustion on the day of the tensiomyography and biopsy collection.

For study 2, biopsies and tensiomyographic data were collected from 17 participants at least $24 \mathrm{~h}$ before or after the competition, without any high-intensity exercise at the day of testing. In 14 participants, tensiomyographic measurements were performed before, and in 3 participants, $24 \mathrm{~h}$ after the biopsies were taken. Because the data collected from these three participants did not deviate from those obtained from other participants, all data were pooled for further analysis.

Tensiomyography measurement. Measurements were performed supine at rest at a knee angle set at $30^{\circ}$ knee flexion, where $0^{\circ}$ represents the extended joint. The oscillations of the muscle belly in response to an electrically induced twitch were recorded at the skin surface using a sensitive digital displacement sensor (TMG-BMC, Ljubljana, Slovenia). The sensor was perpendicular to the skin overlying the muscle belly, at $30 \%$ of femur length above the patella on the lateral side of the vastus lateralis muscle (Fig. 1) as recommended in the anatomic guide for electromyographers (31). We used a single monophasic 1-ms pulse that elicited the maximal vastus lateralis twitch response on the displacement sensor. The rounded (5-cm diameter) self-adhesive cathode and anode (Axelgaard, Aarhus, Denmark) were set on the vastus lateralis $5 \mathrm{~cm}$ distally and $5 \mathrm{~cm}$ proximally to the measuring point,

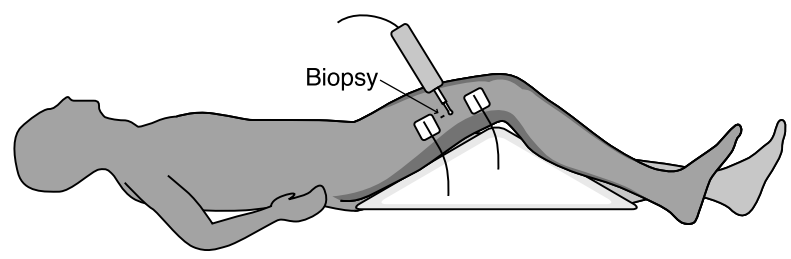

FIGURE 1-Schematic representation of the tensiomyographic measuring setup with marked biopsy spot at $40 \%$ (from the distal end) of the connecting line between the lateral knee cleft and the spina iliaca anterior superior. 
respectively. If needed, the measuring point and electrode positions were adjusted to obtain the maximal displacement amplitude (Dm). The stimulation current at the start was just above threshold and was then gradually increased until the amplitude of the radial twitch displacement did not increase further. Two maximal twitch responses were triggered, recorded, and saved via a parallel computer port. From every twitch response, the $\mathrm{Dm}, \mathrm{Td}, \mathrm{Tc}$, and $\mathrm{Tr}$ were calculated (Fig. 2). At time 0 (total muscle rest at the moment of electrical impulse initiation), an initial displacement was defined. Dm was defined as the peak amplitude in the displacementtime curve of the tensiomyographical twitch response. Td was defined as the time between the electrical stimulus and displacement of the sensor to $10 \%$ of Dm, Tc was the time from $10 \%$ to $90 \%$ of Dm reached, and $\operatorname{Tr}$ was the time from $90 \%$ $\mathrm{Dm}$ to decline to one-half of the Dm in the relaxation phase (Fig. 2). The average value of these parameters in the two twitches was used for further analysis.

Muscle biopsy and \%MHC determination. A conchotome was used to obtain a percutaneous biopsy of the vastus lateralis at $40 \%$ midways between the knee joint cleft and the spina iliaca anterior superior. Thereto, the biopsy site was anaesthetized with $2 \%$ lidocaine and sterilized. After local anesthesia, a small incision was made, and approximately $100 \mathrm{mg}$ of muscle tissue was harvested. The biopsy was immediately placed on a cork with the long axis of the fibers perpendicular to the plane of the cork. The biopsy was then frozen in liquid nitrogen with vigorous shaking to prevent the development of ice crystals. The samples were stored at $-80^{\circ} \mathrm{C}$.

The \%MHC was determined as described previously (13). In a cryostat, $10-\mu \mathrm{m}$ cross-sections were collected in a centrifuge tube and dissolved in a sample buffer (three sections in $100 \mu \mathrm{L}$ ) and boiled for $2 \mathrm{~min}$ to denature the proteins. Twelve-microliter samples were loaded on sodium dodecyl sulfate polyacrylamide gel run at $15^{\circ} \mathrm{C}, 120 \mathrm{~V}$ for $27 \mathrm{~h}$. The stacking gel contained $4 \%$ acrylamide, and the
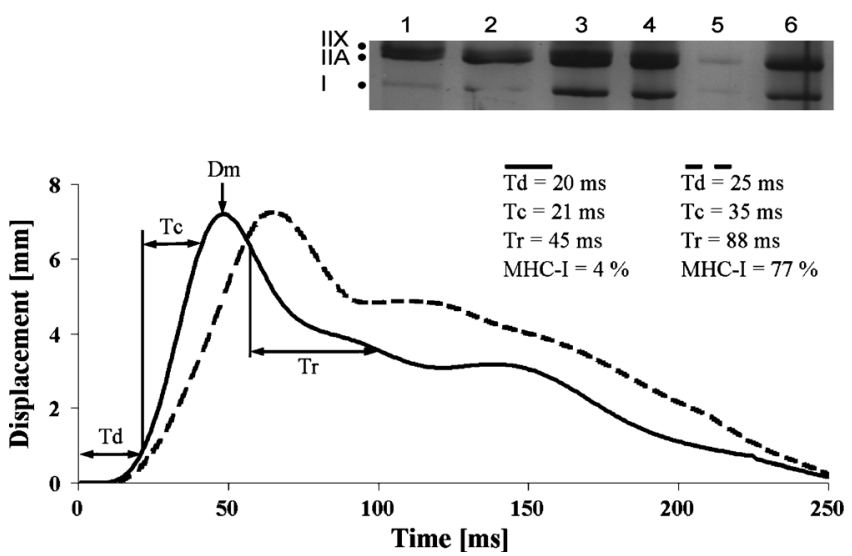

FIGURE 2-Radial twitch responses with shortest (solid line) and longest (dashed line) Tc. For further explanation, see the text. Above are lanes of four participants, with lanes 1 and 2 of muscle biopsies with only $4 \%$ type I MHC. All other lanes are muscles with more than $20 \%$ type I MHC.
TABLE 1. Summary of average MHC proportion and tensiomyographic parameters in the human vastus lateralis muscle.

\begin{tabular}{lc}
\hline & Total \\
\hline Tensiomyographic data & \\
Maximal displacement (mm) & $7.2 \pm 2.5$ \\
Td (ms) & $22.3 \pm 1.2$ \\
TC (ms) & $25.9 \pm 3.8$ \\
Tr (ms) & $92.7 \pm 32.4$ \\
MHC proportion data (\%) & \\
MHC-I & $31.1 \pm 16.9$ \\
MHC-II & $68.9 \pm 16.9$ \\
MHC-IIA & $47.5 \pm 15.7$ \\
MHC-IIX & $21.5 \pm 18.1$ \\
\hline
\end{tabular}

separating gel had $7 \%$ acrylamide with both containing $30 \%$ glycerol. After the run, the gels were stained with the Silver Stain Plus kit following the instructions of the manufacturer (Bio-Rad Laboratories, United Kingdom). Bands were identified on the basis of the migration distance (Fig. 2), and relative quantities were determined with the Quantity One software (Bio-Rad Laboratories).

Statistical analysis. All data are expressed as means \pm SD. For all parameters, a normal distribution was checked with the Shapiro-Wilk test for small samples. The effects of age and gender on tensiomyographic parameters and MHC composition were analyzed with a two-way ANOVA. Univariate and multivariate linear regression analyses were used to determine relationships between \%MHC-I and tensiomyographic parameters. The Pearson correlation coefficients $(r$ and $R$ ) were expressed to present the size of (bivariate and multiple) regression. SE of the estimate was presented together with the $95 \%$ confidence interval for the multiple regression model. Statistical significance was accepted at $P<0.05$ level.

The aim of our study was to evaluate the estimation of the \%MHC-I from the radial twitch Tc, Td, and $\mathrm{Tr}$ in the human vastus lateralis.

\section{RESULTS}

Descriptive analysis. The average \%MHC and tensiomyographic data are given in Table 1. All observed variables passed the Shapiro-Wilk normality test. There was no substantial effect of age or gender on any of the parameters studied.

Tensiomyographic data. Figure 2 shows two tensiomyographic responses with the shortest $(20.9 \mathrm{~ms})$ and longest $(35.1 \mathrm{~ms}) \mathrm{Tc}$, which had also the lowest $(4.3 \%)$ and largest (76.9\%) \%MHC-I, respectively. The mean and SD of tensiomyographic $\mathrm{Tc}$ are as expected for a muscle of $31.1 \%$ MHC-I (10). We focused on the proportion of type I MHC because fibers of this type are the slowest, and the difference in maximal shortening between type I and IIa fibers is much larger than that between the type II subtypes (15).

Linear regression of \%MHC-I versus tensiomyographic parameters. Univariate regression models revealed that $\mathrm{Td}, \mathrm{Tc}$, and $\mathrm{Tr}$ all correlated significantly with 
\%MHC-I at $r=0.612(P=0.001), 0.878(P<0.001)$, and $0.669(P<0.001)$, respectively (Fig. 3). We also found significant, though weaker, correlations between each of the
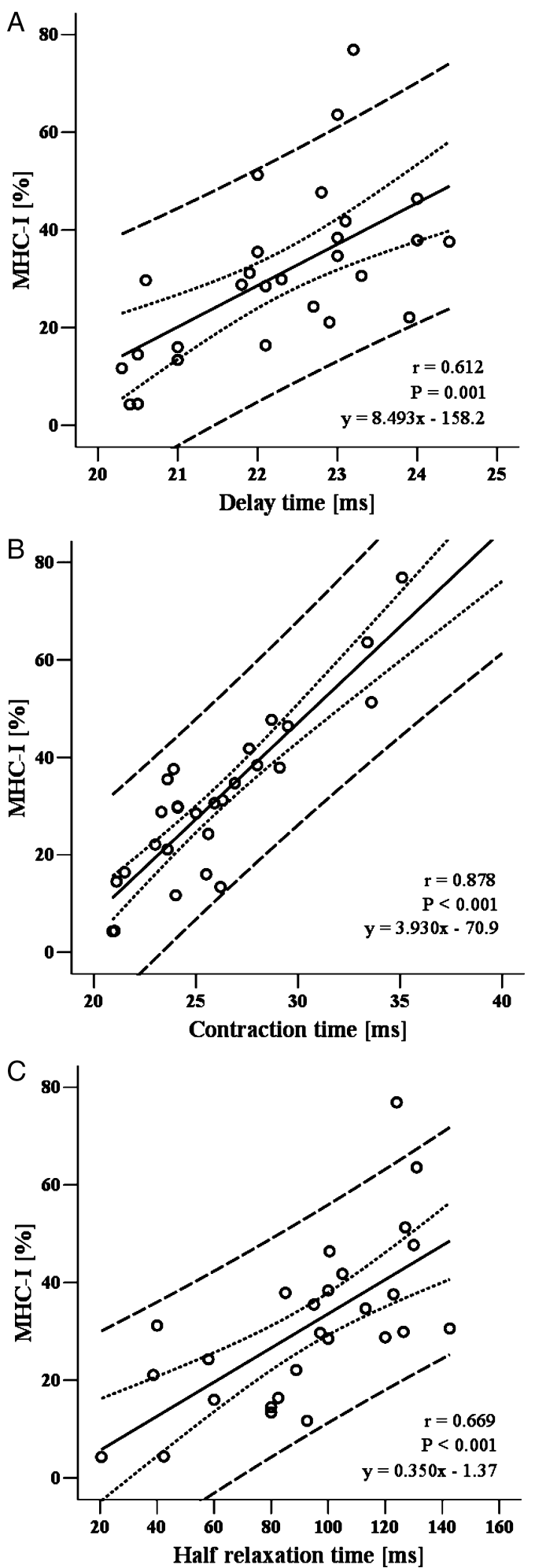

FIGURE 3-Univariate regression models between the radial twitch Td (A), Tc (B), and Tr (C) measured by tensiomyography and the $\%$ MHC-I isoform. Straight, dotted, and dashed lines represent fitted linear models, $95 \%$ confidence intervals, and $95 \%$ estimate intervals, respectively.
TABLE 2. Multiple linear regression analysis of tensiomyographic parameters as predictors of \%MHC-I.

\begin{tabular}{lcrccc}
\hline Predictor & $\boldsymbol{B}$ & SE $\boldsymbol{B}$ & $\boldsymbol{\beta}$ & Part $\boldsymbol{R}$ & $\boldsymbol{P}$ \\
\hline Constant & $-121.023 \%$ & 23.38 & - & - & - \\
Td & $2.829 \% \cdot \mathrm{ms}^{-1}$ & 1.22 & 0.20 & 0.17 & 0.029 \\
Tc & $2.980 \% \cdot \mathrm{ms}^{-1}$ & 0.40 & 0.67 & 0.55 & $<0.001$ \\
Tr & $0.127 \% \cdot \mathrm{ms}^{-1}$ & 0.05 & 0.24 & 0.20 & 0.014 \\
\hline
\end{tabular}

$B$, unstandardized coefficient; $\beta$, standardized coefficient.

three radial twitch parameters, $r<0.50$. Figure 3 displays the linear regression models of $\mathrm{Td}$, $\mathrm{Tc}$, and $\mathrm{Tr}$ with the $\%$ MHC-I.

Results of the multiple linear regression analysis are presented in Table 2. Variables entered were Td, Tc, and Tr as predictors of \%MHC-I. All three parameters contributed positively and significantly to the prediction of the \%MHC-I (Fig. 3). Figure 4 shows that the \%MHC-I predicted with the model correlated significantly with the measured \%MHC-I $(R=0.933, P<0.001)$. SE of the estimate was $6.08 \%$ with $95 \%$ estimate intervals of the sample mean $(19.15 \%$, $42.97 \%$ ). The multiple regression model leaves $12.9 \%$ of the $\% \mathrm{MHC}-\mathrm{I}$ variance unexplained.

\section{DISCUSSION}

To the best of our knowledge, there is currently no easy and relatively inexpensive noninvasive method available that provides a good estimate of the fiber type composition of a human skeletal muscle. In a previous study, Dahmane et al. (10) used tensiomyographic measurement of Tc in seven skeletal muscles but not in the vastus lateralis and compared it with the fiber type composition in corresponding cadaveric muscles. In the present study, we correlated the tensiomyographic data with the MHC composition of the very

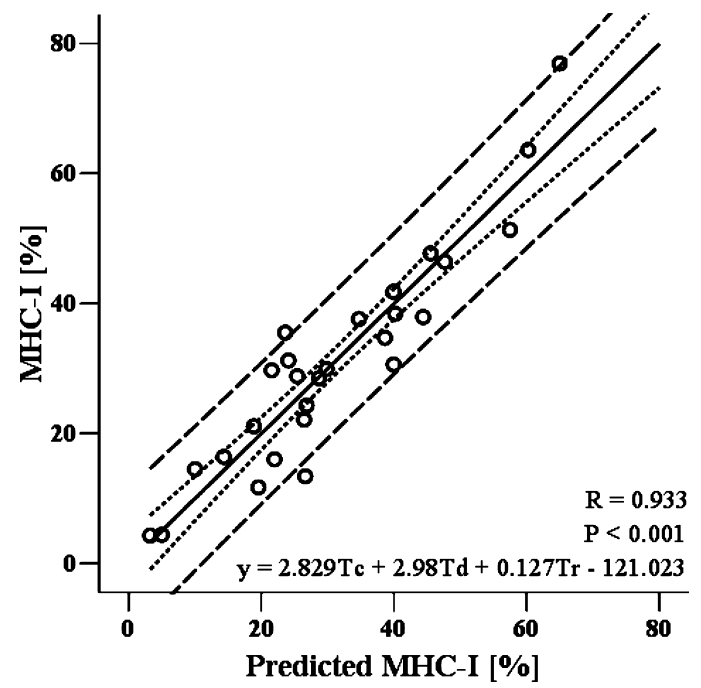

FIGURE 4-Multivariate regression model between the estimated proportion of MHC-I isoform from radial twitch Td, Tc, and Tr and the actual \%MHC-I isoform. Solid, dotted, and dashed lines represent fitted linear models, $95 \%$ confidence intervals, and $95 \%$ estimate intervals, respectively. 
same muscle. The results of our present study indicate that the relatively simple method of tensiomyography can indeed be used to estimate the \%MHC-I in human vastus lateralis muscle noninvasively.

Individual mammalian motor units can be differentiated in terms of the velocities of their isometric force twitches into "fast" and "slow" types using isolated muscle preparations (5-9). Tensiomyography does not measure muscle force but analyzes muscle belly displacement during an isometric twitch. The Tc was measured as reported in previous tensiomyography studies. In comparison with the Tc values reported by Dahmane et al. (10) in seven muscles, the Tc in the vastus lateralis muscle $(25.9 \pm 3.8 \mathrm{~ms})$ is comparable to the extensor digitorum and triceps brachii muscles. For the three tensiomyographic parameters included in this study, the previously reported short-term reproducibility is high $(0.86 \leq$ intraclass correlation coefficient $\leq 0.97)$ with a normalized SE of the measurement $<2 \%$ (20).

The radial twitch response of the muscle belly resembles the time course in the force/torque twitch response (36). However, the radial twitch response reaches a maximal response at lower muscle forces (radial time to peak $<$ force/ torque time to peak), and the effects of joint mechanics, muscle cross talk, elasticity, and damping during muscle movement are smaller $(27,36)$. Tc was the best estimator of MHC composition, followed by $\mathrm{Tr}$ and $\mathrm{Td}$. Although these three parameters describe three time-distinct phases of muscle twitch contraction, they were all correlated.

Muscle fiber conduction velocity in the human vastus lateralis varies between 4.13 and $5.20 \mathrm{~m} \cdot \mathrm{s}^{-1}$ (35) and represents a substantial part of the electromechanical delay after twitch stimulation. The muscle fiber conduction velocity is slower in slow-twitch than in fast-twitch fibers $(1,35)$ and has been used to estimate the relative area of fast-twitch fibers within a muscle (35). Differences in conduction velocity may thus partly explain the correlation between $\mathrm{Td}$ and the \%MHC-I we observed.

Muscle contraction is due to cyclic interactions of myosin and actin (cross-bridge cycles). In the sliding filament model, the $\mathrm{Ca}^{2+}$ is secreted from the sarcoplasmic reticulum. $\mathrm{Ca}^{2+}$ binds to troponin $\mathrm{C}$ and enables binding of both contractile elements in a cross-bridge attachment. In the depolarization phase, the $\mathrm{Ca}^{2+}$ is released from troponin $\mathrm{C}$, triggering a cross-bridge detachment, when $\mathrm{Ca}^{2+}$ is sequestrated by the sarcoplasmic reticulum. Twitch Tc and Tr are dependent on the rate of $\mathrm{Ca}^{2+}$ release from and sequestration by the sarcoplasmic reticulum, respectively. During isometric muscle contraction, sarcomere length shortens, although not the muscle fiber length. Shortening of the sarcomeres results in tension development at both ends of the muscle fibers. Therefore, the tension is a function of the number of cross-bridges coupled during muscle contraction. During a single twitch, the cross-bridges remain active as long as enough cytosolic $\mathrm{Ca}^{2+}$ is available. The tension produced by sarcomeres is transmitted to bone through series elastic components (connective tissue, titin, and ten- dons). A study that examined the time course of the length and velocity of muscle fascicles and tendinous tissues during twitch isometric stimulation found that the fascicle and tendinous tissue lengths and external torque showed a 10- to 30-ms delay of each onset (25). It could be concluded that mechanical interactions between fascicles and tendinous tissues are significant determinants of twitch force and time characteristics. However, just a few have analyzed the transversal dimension of muscle geometry changes (41). They found a linear correlation between longitudinal and transversal muscle fiber strain, where the slope was -0.98 , indicating that all changes of muscle length are accounted for by muscle fiber transverse strain. This finding directly validates tensiomyographic displacement measurements of isometric muscle contraction.

Clearly, the Tc and relaxation time do not entirely estimate the \%MHC-I in the muscle. It should be noted that a biopsy is necessarily a small sample of the muscle as a whole, and it is possible that the biopsy is not an accurate representation of the muscle as a whole. This was evaluated in the vastus lateralis several times, and authors report variation between duplicate biopsies ranging from $6.2 \%$ to $12.3 \%$, concerning \%type I fibers (2) and therefore propose more biopsy sampling from different depths of the muscle (21). On the other hand, tensiomiyography measures the maximal mechanical twitch response of the underlying muscle fibers. In addition, in-series elasticity of the muscle fibers and connective tissues has an effect on the skeletal muscle mechanical response. Nevertheless, this effect should further improve the correlation between relaxation time and \%MHC-I because fast fibers have been reported to store more elastic energy than slow fibers (3), which may further enhance the speed of relaxation of fast fibers.

A similar method and apparatus to the ones used in the current study for in vivo determination of muscle fiber were patented by Moss (24) in 1987, and using multiple regression models, this author observed a similar correlation between time parameters of a twitch contraction and $\% \mathrm{MHC}-\mathrm{I}$ ( $R$ was from 0.911 to 0.948 ) as in the present study $(R=0.933)$. The system and way of measurement were, however, much more complex than our system. He used in his model the stimulation current amplitude, electrical potential of the muscle, tension curve with six time parameters and three amplitudes, as well as the measurements of force and electromyography, whereas we recorded only the $\mathrm{Td}$, Tc, and Tr of the radial twitch response.

In conclusion, tensiomyography is a simple-to-use, noninvasive, and selective method for the determination of skeletal muscle contractile parameters. The method could be easily applied in superficial human muscles. We found that the $\mathrm{Td}, \mathrm{Tc}$, and $\mathrm{Tr}$ correlated significantly with the $\% \mathrm{MHC}-\mathrm{I}$ in the human vastus lateralis muscle. Our finding suggests that this simple noninvasive method could be used for valid estimation of the \%MHC-I in the human vastus lateralis muscle and most likely also in other human muscles. Our 
study, like most studies with biopsies, included a relatively small sample size; however, the age range was high. That was the compromise we took between internal and external validity control. In the future, more such cross-validity studies should be encouraged to test the robustness of the derived prediction equation.

This study was partly funded by the Osteoporosis and Muscle Atrophy Group of the Italian Space Agency. The National Institutes of Health, Wellcome Trust, Howard Hughes Medical Institute, and other(s) did not fund the authors in any phase of the preparation of this work.

\section{REFERENCES}

1. Barry DT, Geiringer SR, Ball RD. Acoustic myography: a noninvasive monitor of motor unit fatigue. Muscle Nerve. 1985;8(3): 189-94.

2. Blomstrand E, Ekblom B. Needle biopsy technique for fibre type determination in human skeletal muscle - a methodological study. Acta Physiol Scand. 1982;116(4):437-42.

3. Bosco C, Tihanyi J, Komi PV, Fekete G, Apor P. Store and recoil of elastic energy in slow and fast types of human skeletal muscles. Acta Physiol Scand. 1982;116:343-9.

4. Buchtal F, Schmalbruch H. Contraction times and fibre types in intact human muscle. Acta Physiol Scand. 1970;79(4):435-52

5. Buller AJ, Eccles JC, Eccles RM. Differentiation of fast and slow muscles in the cat hind limb. J Physiol. 1960;150:399-416.

6. Bülow PM, Norregaard J, Danneskoild-Samsøe B, Mehlsen J. Twitch interpolation technique in testing of maximal muscle strength: influence of potentiation, force level, stimulus intensity, and preload. Eur J Appl Physiol Occup Physiol. 1993;67(5):462-7.

7. Burke RE, Levine DN, Zajac FE 3rd. Mammalian motor units: physiological-histochemical correlation in three types in cat gastrocnemius. Science. 1971;174(10):709-12.

8. Canaday PG, Fay FS. An ultrasensitive isometric force transducer for single smooth muscle cell mechanics. J Appl Physiol. 1976; 40(2):243-6

9. Close RI. Dynamic properties of mammalian skeletal muscle. Physiol Rev. 1972;52(1):129-7.

10. Dahmane R, Valenčič V, Knez N, Eržen I. Evaluation of the ability to make non-invasive estimation of muscle contractile properties on the basis of the muscle belly response. Med Biol Eng Comput. 2000;39(1):51-5.

11. Degens H, Larsson L. Application of skinned single muscle fibres to determine myofilament function in ageing and disease. $J$ Musculoskelet Neuronal Interact. 2007;7(1):56-61.

12. Degens H, Sanchez Horneros JM, Heijdra YF, Dekhuijzen PN, Hopman MT. Skeletal muscle contractility is preserved in COPD patients with normal fat-free mass. Acta Physiol Scand. 2005; 184(3):235-42.

13. Degens H, Soop M, Höök P, Ljungqvist O, Larsson L. Postoperative effects on insulin resistance and specific tension of single human skeletal muscle fibres. Clin Sci (Lond). 1999;97(4): 449-55.

14. Gilliver SF, Degens H, Rittweger J, Sargeant AJ, Jones DA. Variation in the determinants of power of chemically skinned human muscle fibres. Exp Physiol. 2009;94(10):1070-8.

15. Harridge SD, Bottinelli R, Canepari M, et al. Whole-muscle and single-fibre contractile properties and myosin heavy chain isoforms in humans. Pflugers Arch. 1996;432(5):913-20.

16. Hill AV. The heat of shortening and the dynamic constants of muscle. Proc R Soc Lond B Biol Sci. 1938;126(843):136-95.

17. Hill AV. The plateau of full activity during a muscle twitch. Proc $R$ Soc Lond B Biol Sci. 1953;141(905):498-503.

18. Huijing PA, Baan GC. Extramuscular myofascial force transmis-
The authors thank the Orthopedic Hospital of Valdoltra and the organizing committee of the 16th European Veterans Athletic Championships in Ljubljana, Slovenia, for helping them in conducting experiments in their facilities. The authors also thank Prof. P.E. di Prampero (University of Udine, Italy) and Prof. G. Biolo (University of Trieste, Italy) for supporting this study and other participants in the Valdoltra 2007 bed rest and European Veterans Athletic Championships 2008 studies. The authors also thank the TMG-BMC, Ltd., a tensiomyography patent-holding institution, for supporting them with a tensiomyographic device. Finally, the authors thank the study participants for their willingness to participate in a study of such magnitude.

The results of the present study do not constitute endorsement by American College of Sports Medicine.

sion within the rat anterior tibial compartment: proximo-distal differences in muscle force. Acta Physiol Scand. 2001;173(3): 297-311

19. Kohn TA, Essen-Gustavsson B, Myburgh KH. Exercise pattern influences skeletal muscle hybrid fibers of runners and nonrunners. Med Sci Sports Exerc. 2007;39(11):1977-84.

20. Križaj D, Šimunič B, Žagar T. Short-term repeatability of parameters extracted from radial displacement of muscle belly. $J$ Electromyogr Kinesiol. 2008;18(4):645-51.

21. Lexell J, Taylor C, Sjöström M. Analysis of sampling errors in biopsy techniques using data from whole muscle cross sections. $J$ Appl Physiol. 1985;59(4):1228-35.

22. Maton B, Petitjean M, Cnockaert JC. Phonomyogram and electromyogram relationships with isometric force reinvestigated in man. Eur J Appl Physiol Occup Physiol. 1990;60(3):194-201.

23. Moritani T. Motor unit and motoneurone excitability during explosive movement. In: Komi PV, editor. Strength and Power in Sport. Oxford (UK): Blackwell Scientific Publications; 2005. p. $27-49$.

24. Moss CL, inventor. Method and apparatus for in vivo determination of muscle fiber. United States patent US 4,688,581. 1987 Aug 25. p. 11

25. Oda T, Himeno R, Hay DC, et al. In vivo behavior of muscle fascicles and tendinous tissues in human tibialis anterior muscle during twitch contraction. J Biomech. 2007;40(14):3114-20.

26. Orizio C. Comments on the letter "accelerometer and mechanomyogram." J Biomech. 2002;35(3):385.

27. Orizio C, Baratta R, Zhou B, Solomonow M, Veicsteinas A. Force and surface mechanomyogram relationship in cat gastrocnemius. $J$ Electromyogr Kinesiol. 1999;9(2):131-40.

28. Orizio C, Baratta RV, Zhou BH, Solomonow M, Veicsteinas A. Force and surface mechanomyogram frequency responses in cat gastrocnemius. J Biomech. 2000;33(4):427-33.

29. Orizio C, Liberati D, Locatelli C, DeGrandis D, Veicsteinas A. Surface mechanomyogram reflects muscle fibres twitches summation. J Biomech. 1996;29(4):475-81.

30. Orizio C, Veicsteinas A. Soundmyogram analysis during sustained maximal voluntary contraction in sprinters and long distance runners. Int J Sports Med. 1992;13(8):594-9.

31. Perotto AO, Delagi EF, Iazzetti J, Morrison D. Anatomic Guide for the Electromyographer: the Limbs. Springfield (IL): Charles C. Thomas; 2005. p. 235-8.

32. Petrofsky JS, Lind AR. Isometric endurance in fast and slow muscles in the cat. Am J Physiol. 1979;236(5):C185-91.

33. Pišot R, Narici MV, Simunič B, et al. Whole muscle contractile parameters and thickness loss during 35-day bed-rest. Eur J Appl Physiol. 2008;104(2):409-14.

34. Prasartwuth O, Allen TJ, Butler JE, Gandevia SC, Taylor JL. Length-dependent changes in voluntary activation, maximum voluntary torque and twitch responses after eccentric damage in humans. J Physiol. 2006;571(Pt 1):243-52. 
35. Sadoyama T, Masuda T, Miyata H, Katsuta S. Fibre conduction velocity and fibre composition in human vastus lateralis. Eur $J$ Appl Physiol Occup Physiol. 1988;57(6):767-71.

36. Šmunič B, Križaj D, Narici M, Pišot R. Twitch parameters in transversal and longitudinal biceps brachii response. Ann Kin. 2010;1:61-80.

37. Stevens JC, Dickinson V, Jones NB. Mechanical properties of human skeletal muscle from in vitro studies of biopsies. Med Biol Eng Comput. 1980;18(1):1-9.

38. Swammerdam J. The Book of Nature II. London (UK): Seyffert; 1758. p. $122-32$.

39. Terzis G, Spengos K, Kavouras S, Manta P, Georgiadis G. Muscle fibre type composition and body composition in hammer throwers. J Sport Sci Med. 2010;9:104-109.
40. Valenčič V, Knez N. Measuring of skeletal muscles dynamic properties. Artif Organs. 1997;21:240-2.

41. van Donkelaar CC, Willems PJB, Muijtjens AMM, Drost MR. Skeletal muscle transverse strain during isometric contraction at different lengths. J Biomech. 1999;32:755-62.

42. Westerblad H, Allen DG. The contribution of $\left[\mathrm{Ca}^{2+}\right] \mathrm{i}$ to the slowing of relaxation in fatigued single fibres from mouse skeletal muscle. J Physiol. 1993;468:729-40.

43. Wong YM. Letter to the editor: accelerometer and mechanomyogram. J Biomech. 2001;34(4):557.

44. Zhang Y, Frank CB, Rangayyan RM, Bell GD. A comparative study of simultaneous vibromyography and electromyography with active human quadriceps. IEEE Trans Biomed Eng. 1992; 39(10):1045-52. 